\title{
A Hybrid PAPR Reduction Scheme for Optical Wireless OFDM Communication Systems
}

\author{
${ }^{1,2}$ Ayad A. Abdulkafi ${ }^{*},{ }^{1}$ Mohamad Yusoff Alias, ${ }^{1}$ Yaseein Soubhi Hussein, ${ }^{3}$ Nazaruddin Omar, \\ and ${ }^{3}$ Mohd Kamarulzamin Bin Salleh \\ ${ }^{1}$ Faculty of Engineering, Multimedia University, Persiaran Multimedia, Cyberjaya, Selangor, Malaysia \\ ${ }^{2}$ Electrical Engineering Department, Tikrit University, Salahaddin, Iraq \\ Fige-mail: al.ayad@yahoo.com, yusoff@mmu.edu.my, yaseein@ieee.org] \\ ${ }^{3}$ TM Research \& Development Sdn Bhd, Innovation Center, Lingkaran Teknokrat Timur, 63000 Cyberjaya \\ Selangor, Malaysia \\ [e-mail: nazar@tmrnd.com.my, kamarulzamin@tmrnd.com.my] \\ *Corresponding author: Ayad A. Abdulkafi
}

Received June 7, 2017; revised September 23, 2017; accepted October 22, 2017;

published March 31, 2018

\begin{abstract}
This paper proposes a new hybrid scheme to decrease the high peak to average power ratio (PAPR) of optical orthogonal frequency division multiplexing (OFDM) signals in visible light communication (VLC) systems. The PAPR causes nonlinear signal distortions and high power requirements for the VLC transmitter (light emitting diode, LED). The proposed method is applicable for both direct current-biased optical OFDM (DCO-OFDM) and asymmetrically clipped optical OFDM (ACO-OFDM). In the proposed scheme, the PTS method is firstly modified to fit both optical OFDM approaches transmission and then combined with signal clipping method for further PAPR reduction and bit error rate (BER) improvement of the VLC system. The performance of hybrid scheme has been evaluated and compared with the original OFDM based VLC system, conventional PTS and clipping methods. The results show that the hybrid scheme outperforms other methods in terms of both the PAPR reduction and BER performance.
\end{abstract}

Keywords: OFDM, VLC, PAPR, PTS, Clipping 


\section{Introduction}

Visible light communication (VLC) has attracted an increasing interest in the field of next generation wireless transmission technologies. According to recent market research report, the global VLC market size is expected to grow at compound annual growth rate (CAGR) of $62.9 \%$ between 2017 and 2022 [1]. The main property of VLC system is its ability to provide illumination and communication simultaneously as well as it supplements the unlicensed spectrum resource. In addition, the enhancements of power efficiency and cost reduction enable light emitting diode (LED) to be deployed in VLC applications. It is possible to use a LED as a transmitter for optical wireless communication (OWC) because it can be switched on and off at a relatively high speed compared to conventional lighting sources. Along with that, Orthogonal frequency division multiplexing (OFDM) offers a high spectral efficiency and it can eliminates the intersymbol interference (ISI) by inserting a guard band, i.e., cyclic prefix (CP). Hence, utilizing the OFDM with VLC system is also a research hotspot [2]-[3] as OFDM is especially suited for an OWC system like VLC. While OFDM offers many advantages for indoor VLC systems, it has a number of disadvantages. The nonlinear characteristic of the LED emitter significantly influences the VLC system's performance that is based on the intensity-modulation direct-detection (IM/DD) along with OFDM modulation. This is because the OFDM signal has predominant high peak-to average power ratio (PAPR) problem. Also, OFDM requires a large linear operational dynamic range because the signal exhibits a Gaussian distribution with a high PAPR. Due to the high PAPR, a system with a limited linear dynamic range results in clipping of the peak amplitudes of the OFDM signal, which introduces additional clipping noise [3]. Our recent works [4]-[5] have discussed the implementation of indoor VLC systems using a direct current-biased optical OFDM (DCO-OFDM) scheme [4] and proposed a new transmission approach for OFDM based VLC in order to increases the bandwidth efficiency [5]. The present work is dedicated to investigate the possible reduction of the PAPR for asymmetrically clipped optical OFDM (ACO-OFDM). The rest of the paper is organized as follows. In Section 2, some related works are reviewed and discussed. In Section 3, we present the optical OFDM approaches in VLC systems. Section 4 presents the system models proposed to evaluate the performance improvements of OFDM based VLC system and PAPR reduction techniques. The simulation results and discussions are presented in Section 5. Finally, Section 6 provides the concluding remarks.

\section{Related Works}

The high PAPR of OFDM signals has emerged as a key challenge for OFDM based VLC systems. Consequently, various techniques have been proposed in literature to overcome this issue such as signal clipping, precoding, selective mapping (SLM) and partial transmit sequence (PTS) techniques. PAPR reduction of VLC-OFDM signals via signal clipping is straightforward and widely used in the literatures due to its simplicity [3]- [8]. Clipping performed by removing the high-power peaks of the signal, without changing its phase by multiplying the signal by a predetermined threshold. The recoverable upper clipping procedure of ACO-OFDM signal clipping in the time domain has been proposed in [7]. However, signal clipping is a nonlinear process that leads to the increase the bit error rate (BER) as well as decreases the spectral efficiency and distorts the OFDM signal. An alternative approach is to use a simpler type of prcoding that consists of multiplying the signal 
by a matrix to reduce the autocorrelation of the input sequence and thus the signal's PAPR. The precoding matrices can be based on the Hadamard transform or the discrete cosine transform [9]-[10]. The work in [11] proposed the using of trellis coding to reduce the large negative peaks of the bipolar signal. However, increasing the required transmission bandwidth of VLC system is the main drawback of these techniques.

Other methods of signal transformation such as the SLM method for reducing the signal peak values in an optical OFDM system has been studied in [12]-[13]. SLM method can statistically enhance the characteristics of the PAPR distribution of the OFDM signal by transmitting a huge amount of overhead in order to properly demodulate data [14]. On the other hand, some techniques are proposed to compensate the effects of PAPR at the receiver. For instance, equation method [15] has investigated the correction of bit-errors caused by clipping distortion which employed to reduce the PAPR in OFDM symbols. A semi-blind error resilient SLM scheme is proposed in [16] to allow error detection and correction in the side information index by utilizing the spread spectrum codes for embedding the side information index in the transmitted signals . Howevere, the main issues of these methods are the high complexity and the transmission of side information for each OFDM symbol to inform the receiver about the selected candidate index [12]. For this, the data transmission efficiency is unavoidably reduced.

Among mentioned methods to reduce PAPR, the PTS method is a distortionless signal scrambling process and provides an effective way for PAPR reduction. It is based on combining signal subblocks which are phase-shifted by constant phase rotation factors. By adjusting the phase factor value, PTS method processes of OFDM signals, selects signals with a minimum PAPR, and then transmits it. It works with an arbitrary number of subcarriers and any modulation scheme [17]. The large computational complexity is the main issue with PTS technique because it requires an exhaustive search over all the combinations of allowed phase factors and due to this, the complexity increases exponentially with respective the number of subblocks. Subblock partitioning also affects the PAPR reduction performance in PTS technique. Moreover, the use of PTS technique has not been fully investigated so far in VLC system and only few researches considered the application of PTS method to reduce the PAPR of OFDM signals in VLC system [18]-[19]. However, earlier works either did take into account the BER performance only [18] or the applicability of PTS for reducing the PAPR is not deeply addressed in VLC systems [19].

This paper proposes a new and hybrid scheme for reducing the PAPR of OFDM signals in VLC system using combined PTS and signal clipping techniques. In the proposed hybrid scheme, the PTS method is modified to be suitable for VLC transmission and then combined with signal clipping method for further improvements in both the PAPR reduction and BER performance of the VLC system.

\section{Optical OFDM Approaches}

In VLC system, the OFDM baseband signal must be real valued and positive because it modulates the optical intensity rather than the amplitude or phase as well as it uses the envelope variation of the OFDM signal. Hence, modulation signals have to be both real and unipolar. However, conventional OFDM signals are both complex and bipolar in nature. It is possible to obtain a real OFDM signal by imposing Hermitian symmetry (HS) in the inverse fast Fourier transform (IFFT) operation during the signal generation step. To achieve a unipolar signal, one can apply a straightforward approach by simply adding a DC-bias current 
to the signal to form the so-called DCO-OFDM scheme. The addition of a constant DC-bias current results in a high-power requirement [2]. Further details on the DCO-OFDM performance in VLC system can be found in [20]. Nevertheless, for indoor VLC systems that are designed for lighting applications, this power constraint is not very significant as the constant DC-bias current is regardless required to provide a constant level of room illumination. The other approach to ensure a unipolar signal is to use the ACO-OFDM [21], in which the signal is clipped at zero and only the positive real parts is being transmitted. This is possible because the OFDM signal is generated in such a way that the positive part is an anti-symmetrical copy of the negative part [21]. This paper mainly focuses on the performance analysis of the VLC system based on ACO-OFDM taking into account its special time domain structure and its better power efficiency when compared to the previous approach.

\section{System Models}

The proposed VLC system model based on an ACO-OFDM scheme considered in this study is illustrated in Fig. 1. The following subsections describe the details of each component of the proposed model.

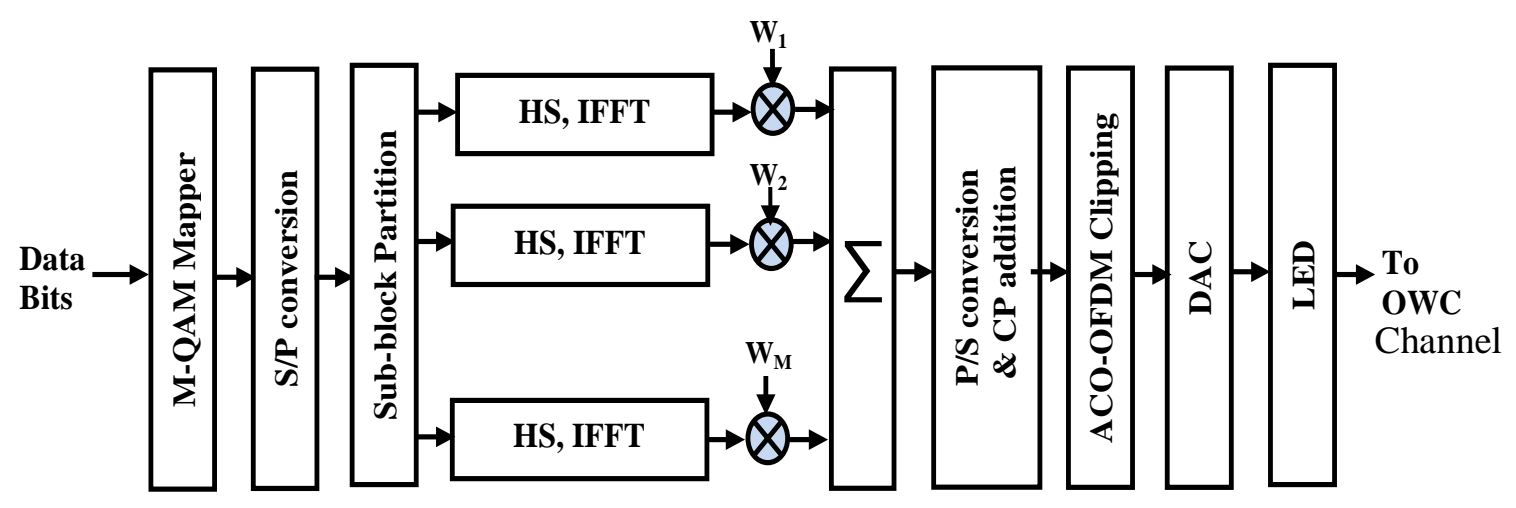

Fig. 1. Block diagram of the proposed scheme for ACO-OFDM based VLC transmitter

\subsection{ACO-OFDM Scheme}

As the IM/DD is employed in ACO-OFDM that modulates the light intensity emitted by the LED, the time-domain transmitted signal must be real and positive. First, the input data bits are mapped according to the considered quadrature amplitude modulation (QAM) constellations. The obtained complex QAM are mapped onto a vector $X_{k}$ after modulating the odd subcarriers only and setting the even subcarriers to zeros as well as imposing the Hermitian symmetry (HS) to the frequency domain subcarriers. The odd frequency and Hermitian constraint together mean that there are only $N / 4$ independent complex input values for an $N$ point IFFT.

$$
X_{k}=\left[X_{0}, X_{1}, X_{2} \ldots X_{N / 2-1}, X_{N / 2}, X_{N / 2-1}^{*}, \ldots X_{2}^{*}, X_{1}^{*}\right]
$$

where all values of $X_{k}=0$ for even $k$ including $X_{0}$ and the $X_{N / 2}$ subcarriers to ensure the HS property whereas the odd indexed subcarriers are mapped with QAM symbols. 


$$
X_{n}=\frac{1}{N} \sum_{k=0}^{N-1} X_{k} e^{j \frac{2 \pi n k}{N}} \quad 0 \leq n \leq N-1
$$

To avoid the induced ISI, a CP is inserted to the real time-domain OFDM symbols which are the result of the IFFT of vector $X_{k}$. The generated bipolar signal, $x_{n}$, is then converted to unipolar through clipping of all negative values at zero before modulating the LED.

$$
x_{n}= \begin{cases}x_{n} & x_{n}>0 \\ 0 & x_{n} \leq 0\end{cases}
$$

It is worth to mention that only the odd indexed subcarriers in $X_{k}$ carry the data and hence the obtained time-domain waveform has a half-wave symmetry because the subcarriers of even order are negligible. This symmetry property implies that the same data information is repeated in the first N/2 samples and in the second half of the OFDM symbol. Thus, the unipolar signal can be produced by clipping the negative part without any loss of information. Although clipping causes intermodulation, it does not affect the data-carrying odd indexed subcarriers because it occurs only in the even indexed subcarriers. However, the envelop real-valued time-domain OFDM signal still has a high PAPR. The PAPR is defined as the ratio of the maximum transmitted signal power to the average power, that is

$$
P A P R=10 \log _{10} \frac{\max _{n}{ }^{2}}{E\left[x_{n}^{2}\right]}
$$

where $\max x_{n}^{2}$ is maximum value of the OFDM signal power, E[•] the average of those values [22]. In order to evaluate PAPR reduction, the complementary cumulative distribution function (CCDF) of PAPR is employed. The CCDF of the PAPR is defined as the probability that the PAPR of an OFDM frame exceeds a given reference value and it is the most commonly used measure for illustrating PAPR reduction.

\subsection{PTS Technique Model}

The PTS method partitions $X$ into $M$ disjoint subblocks represented by vectors $\left\{X^{m} \mid \mathrm{m}=0,1 \ldots\right.$ $M-1\}$. Therefore $X$ can be rewritten as

$$
X=\sum_{m=0}^{M-1} X^{m}=\left[X^{0}, X^{1}, X^{2}, \ldots, X^{M-1}\right]^{T}
$$

where $X^{m}$ are the subblocks with equal size that are consecutively placed. To keep the real-valued in time domain of each sub-block, HS has to be guaranteed in partitioning process similar to given in (1). This can be achieved by setting all the elements of $X$ to zeros except the symbols of the corresponding block in the first half of $X$ and its conjugated counterpart in the second half, as demonstrated in [21]. Besides, PTS is easily combined with the corresponding symbol transform scheme, which means dividing data block into sub-blocks or clusters and multiplying appropriate weighting phase factors, $\hat{W}=\left\{\hat{W}^{m}=e^{j \theta m} \mid m=0,1, \ldots, M-1\right\}$ with phase factor $\phi_{m}=0$ or $2 \pi$ commonly, to each sub-block for PAPR reduction [22]. As the time domain 
optical OFDM signal is real-valued, the $\hat{W}^{m}$ values are constrained to be real too, i.e., $\{1,-1\}$, subsequently taking its IFFT to yield [22]

$$
\begin{aligned}
\hat{x} & =\operatorname{IFFT}\left\{\sum_{m=0}^{M-1} \hat{W}^{m} X^{m}\right\} \\
& =\sum_{m=0}^{M-1} \hat{W}^{m} \operatorname{IFFT}\left\{X^{m}\right\} \\
& =\sum_{m=0}^{M-1} \hat{W}^{m} \hat{x}^{m}
\end{aligned}
$$

The weighting coefficients, $W^{m}$ are selected to be the ones among all possible values that minimize the PAPR of the combined signal i.e.

$$
\left[\mathrm{W}^{0}, \ldots, \mathrm{W}^{\mathrm{M}-1}\right]=\underset{\left[W^{0}, \ldots, \ldots, W^{M-1}\right]}{\arg } \min \left(\max _{n=0, \ldots, N-1 \mid}\left|\sum_{m=0}^{M-1} \hat{W}^{m} \hat{x}^{m}[n]\right|\right)
$$

Then, the corresponding time-domain signal after PTS operation, $x_{p t s}$ with the lowest PAPR vector can be written as

$$
X_{p t s}=\sum_{m=0}^{M-1} W^{m} \hat{x}^{m}
$$

\subsection{ACO-OFDM Signal Clipping}

Besides clipping the negative values at zero, an additional signal clipping has to be performed in ACO OFDM in order to fit a high PAPR signal into a limited range corresponding to a specified driving current limits of the LED. It is known that the VLC system is limited by the dynamic range of the LED and the signal outside this range should be clipped [23]. Here, it is assumed that the nonlinear characteristics of LED can be compensated by predistortion process [24]. The LED can be linearized within the interval $[c,-c]$, where $c$ dentotes the pre-specified clipping level. The LED input signal $x_{L E D}$ is obtained from $x_{p t s}$ after clipping process further reduce the PAPR. Let $x_{c l i p}$ denote the clipped version of $x_{p t s}$, which is expressed as

$$
x_{\text {clip }}=\left\{\begin{array}{lc}
c & x_{p t s} \geq c \\
x_{p t s} & -c<x_{p t s}<c \\
-c & x_{p t s} \leq-c
\end{array}\right.
$$

The clipping ratio (CR) is defined as the clipping level normalized by the RMS value $\sigma$, variance of OFDM signal, such that [24] 


$$
C R=\frac{C}{\sigma}
$$

It is worth to mention that, a higher CR corresponds a higher threshold, and hence, the effect of PAPR reduction gets worse, and vise versa. According to the cumulative signal processing and the modifications proposed, the resulting ACO-OFDM signal is ready to be transmitted through LED and would enable the reduction of PAPR significantly as well as improves the BER performance. Due to the background light, the shot noise is assumed to be the dominant source of noise and has been modeled as an additive white Gaussian noise (AWGN) in the simulation. Therefore, the transmitted and the received signal can be expressed as

$$
y_{n}=x_{n}+n_{A W G N}
$$

where $n_{A W G N}$ is an AWGN noise component. At the receiver, after the optical to electrical conversion and CP removal, the received samples of one ACO-OFDM symbol $y_{n}$ are processed using $N$-points complex fast Fourier transform (FFT) operation as given in the following:

$$
Y_{k}=\sum_{n=0}^{N-1} y_{n} e^{-j \frac{2 \pi h k}{N}}
$$

where, the values $Y_{k}, k=0, \ldots, N-1$ are the estimated complex QAM-constellations. Finally, the QAM demodulator is used to estimate the transmitted data.

\section{Numerical Results and Discussion}

In this part, the perfroamnce of ACO-OFDM in VLC system is analysied in term of PAPR reduction and BER perperformance. The simulation results of the previously described methods, i.e. signal clipping, PTS algorithm and the proposed hybrid scheme, are evaluated and compared. The parameters considered for simulation purpose using MATLAB software are shown in Table 1.

Table 1. Simulation Parameters

\begin{tabular}{|l|l|}
\hline \hline Parameter & Specification \\
\hline System & ACO-OFDM based VLC \\
\hline IFFT size $(N)$ & 64 \\
\hline Modulation & 16 QAM \\
\hline Channel & OWC with AWGN \\
\hline PTS subblocks $(M)$ & 4 \\
\hline Weighting phase factor & 2 \\
\hline CP length & 16 \\
\hline CR & $5.5,6,6.5,7 \mathrm{~dB}$ \\
\hline \hline
\end{tabular}




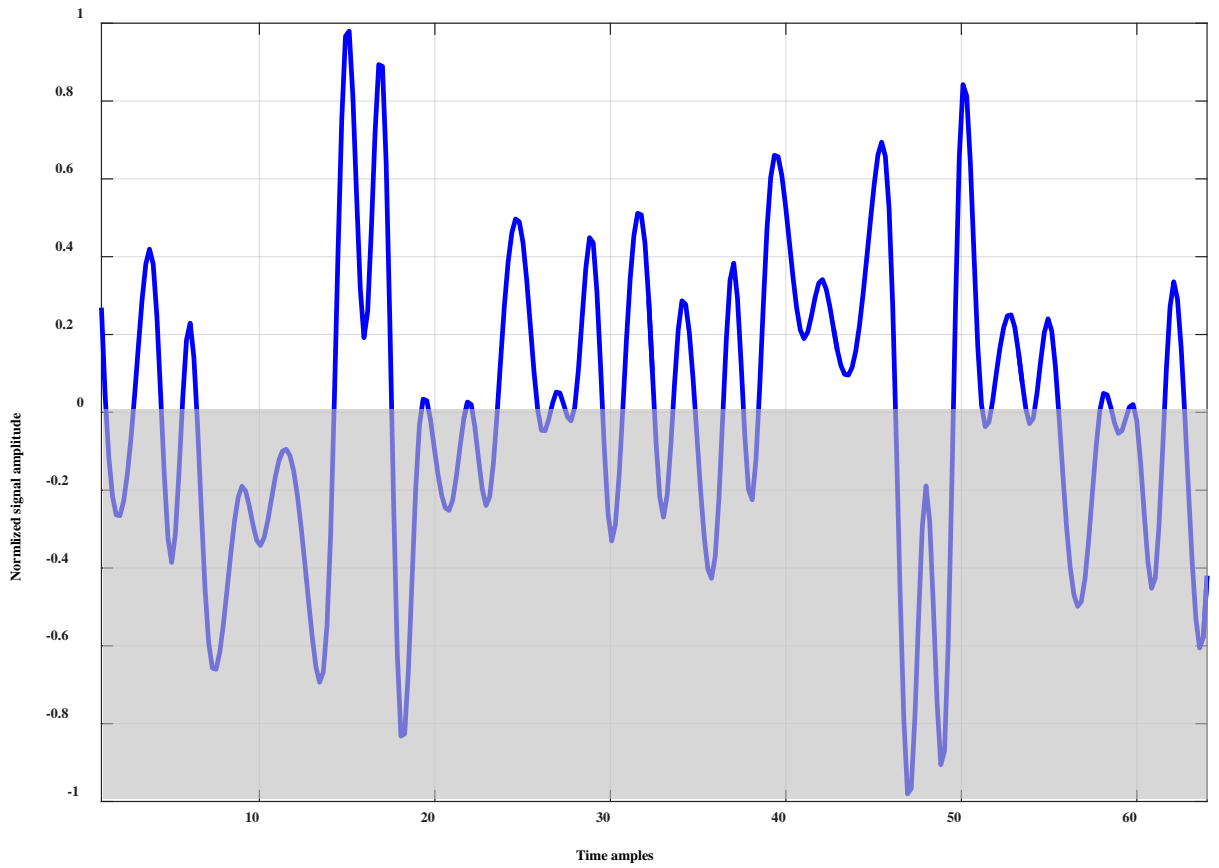

Fig. 2. ACO-OFDM time domain signal. The clipped negative values are illustrated in shaded area

The output waveform of time domain ACO-OFDM signal has a negative part that is a symmetrical copy of its positive part. Clipping the signal at zero will omits this negative part as demonstrated in Fig. 2, thus only the positive part is being transmitted. It can also be seen that the second half of the time domain signal is identical to its inverted first half. This clipped time domain signal is intensity modulated onto the optical carrier. At the receiver, the time domain waveform is FFT-processed and only symbol with odd-indexed subcarrier are passed for symbol detection while the even subcarriers symbol outputs are ignored. The noise resulted from clipping process falls only on the even-numbered sub-carriers, which convey no data. As a consequence, the clipping noise does not deteriorate the QAM symbols on the odd-numbered sub-carriers. The asymmetric clipping reduces the emitted average optical power and hence offers higher signal power efficiency.

However, as only the odd-indexed sub-carriers convey data symbols, ACO-OFDM provides only half the spectral efficiency of DCO-OFDM. To illustrate how the PAPR of VLC OFDM signal can be reduced by using the signal clipping, different clipping ratios (CR) have been considered to show the impact of clipping on both the PAPR reduction and the BER performance. The reductions in PAPR are illustrated using CCDF diagram.The ACO-OFDM signal clipping that takes place at the transmitter in the time domain prior to the DAC stage, as demonstrated in Fig. 1, includes clipping the negative part of signal and the additional clipping to further minimize the remaining high peaks of resulting signal. The signal could be additionally clipped at lower and upper levels according to equation (9). It can be noted from Fig. 3, that the smaller the CR is, the greater the PAPR reduction effect is. For instance, at a CCDF of $10^{-1}$, about $2.5 \mathrm{~dB}$ reduction gain can be obtained by clipping the original ACO-OFDM signal with CR of $5.5 \mathrm{~dB}$. Unfortunately, If the clipping level is low (low CR value), the signal will suffer from distortion caused by clipping while the PAPR will decrease. If the value of CR is high, a clipping distortion decreases while it experiences a high PAPR. 


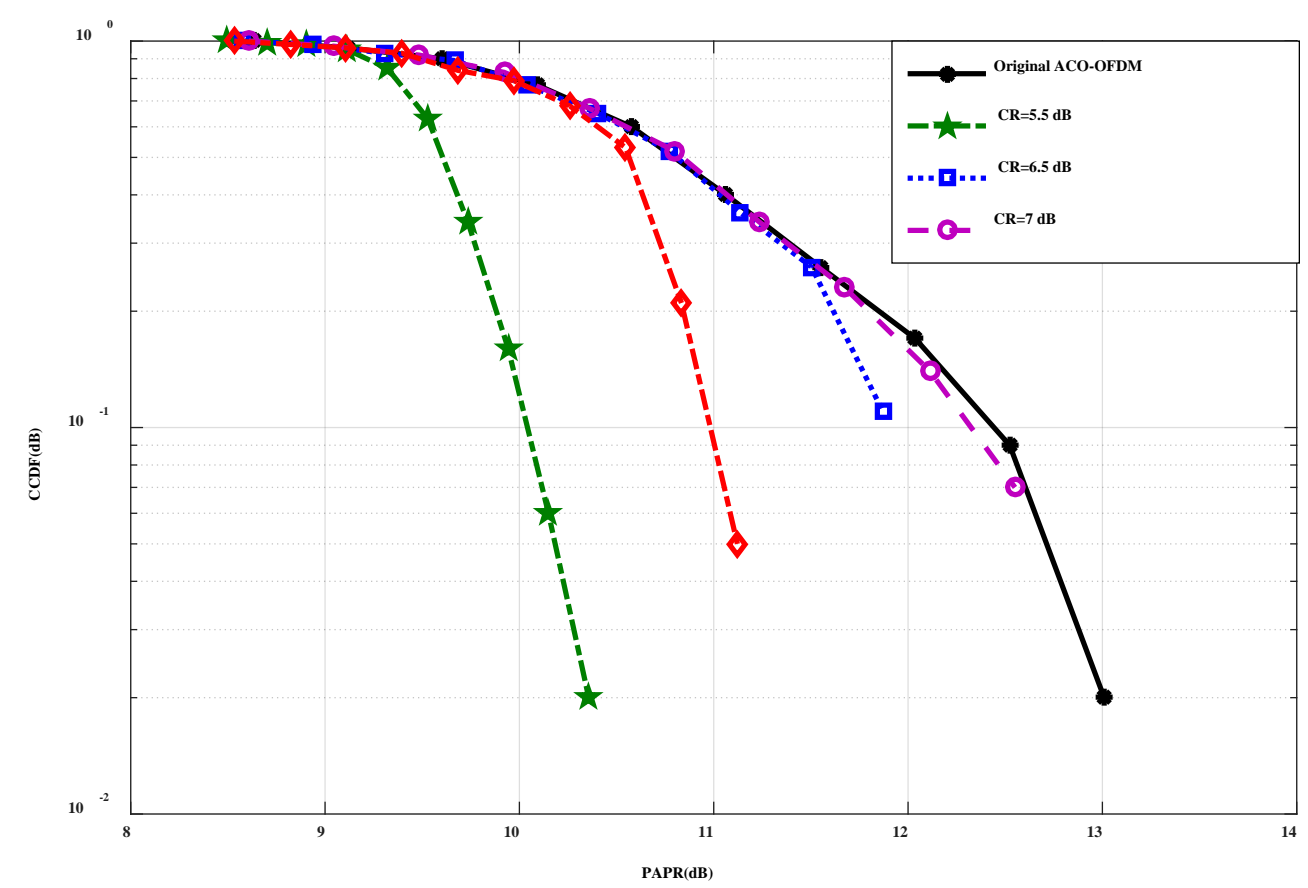

Fig. 3. CCDF of ACO-OFDM signal for for different CR values

However, as the negative part of ACO-OFDM signal is clipped as before to form its structure, the effect of adtional signal clipping is less than on DCO-OFDM but it further reduces the PAPR, as it will be shown later in this work. Although it is possible to attain any desired level of PAPR reduction with signal clipping, this will be at a cost of deterioration in the VLC systems' bit error performance.

Fig. 4 shows the degradation of the BER for different values of CR. The CR and BER are reciprocally related-the smaller the CR, the greater the BER. It can be noted when CR increases, BER always decreases for all SNR values. For example with the same CR of $5.5 \mathrm{~dB}$ that achieve $2.5 \mathrm{~dB}$ PAPR reduction gain, the BER increases from $10^{-1.2}$ (original ACO-OFDM) to $10^{-1}(\mathrm{CR}=5.5 \mathrm{~dB}$ ) for a SNR of $15 \mathrm{~dB}$. From the above simulation results it can be observed that there is a tradeoff between PAPR reduction and the BER performance when employing the signal clipping method. This trade-off relationship between the PAPR reduction and BER performance should be taken into consideration in selecting the proper clipping level in order to achieve better performance of the VLC systems. As aforementioned, PTS approach provides an effective solution for PAPR reductions, however, it results in large computational complexity simultaneously. Compared to PTS, signal clipping provides a simpler way towards a better PAPR performance, whereas results in degradation of the BER performance. Therefore, combining these two methods can achieve better performance in both PAPR and BER performance in VLC system. 


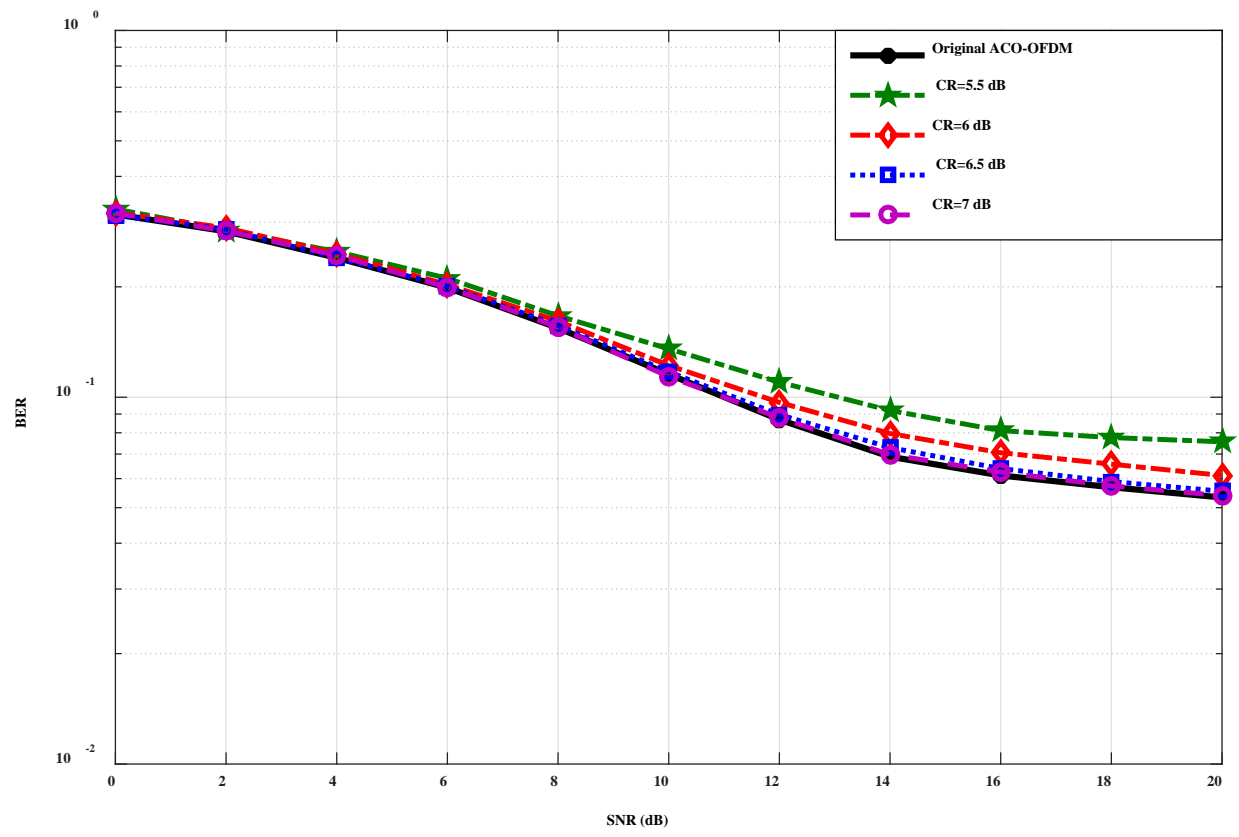

Fig. 4. BER Performance for different CR values

The CCDF performance of the new hybrid scheme which is comprising of the modified PTS method with the signal clipping is shown in Fig. 5. It is obviously seen that the proposed hybrid scheme offers improved PAPR reduction performance compared with other techniques. Clearly, about $8 \mathrm{~dB}$ PAPR reduction gain is obtained from the proposed hybrid scheme when compared with the basic ACO- OFDM signal at a CCDF of $10^{-1}$. The PAPR reduction gain of the proposed method is about $5.5 \mathrm{~dB}$ and $1 \mathrm{~dB}$ compare to the signal clipping (with CR of 5.5 $\mathrm{dB}$ ) and the modified PTS method, respectively.

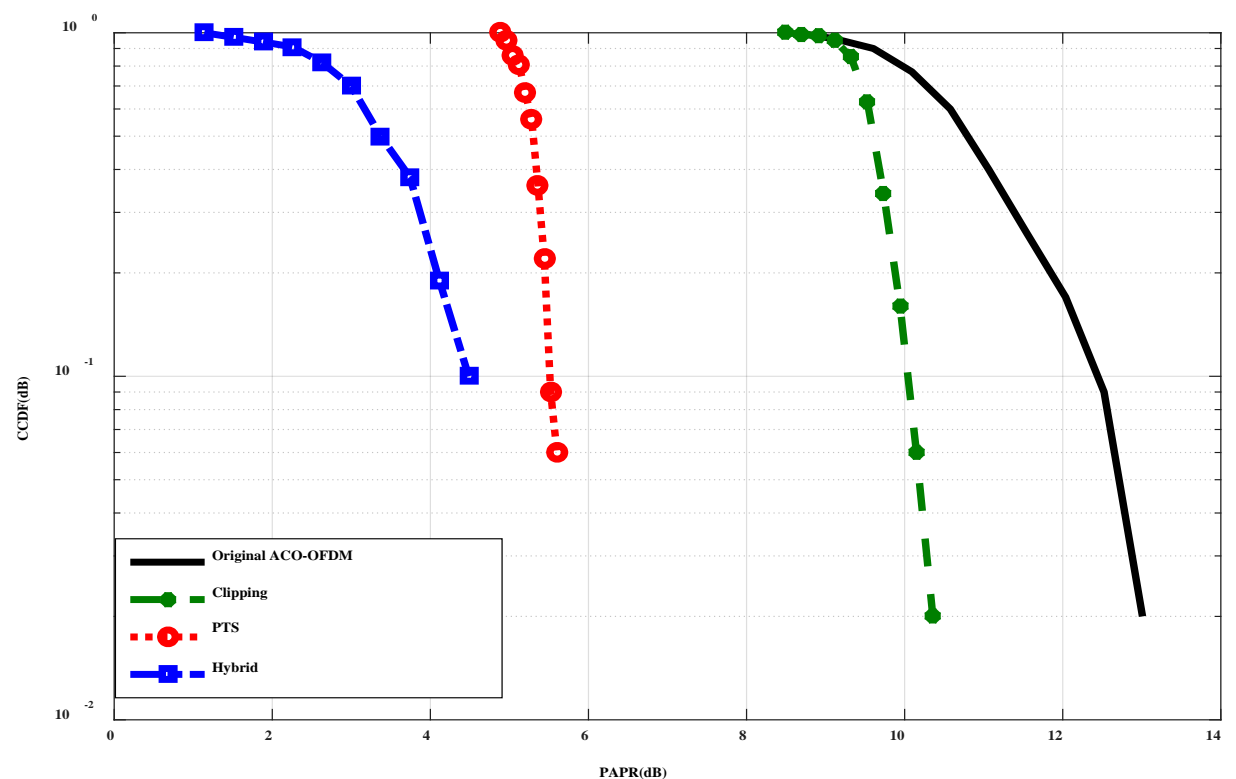

Fig. 5. CCDF performance of the ACO-OFDM signal with different methods 


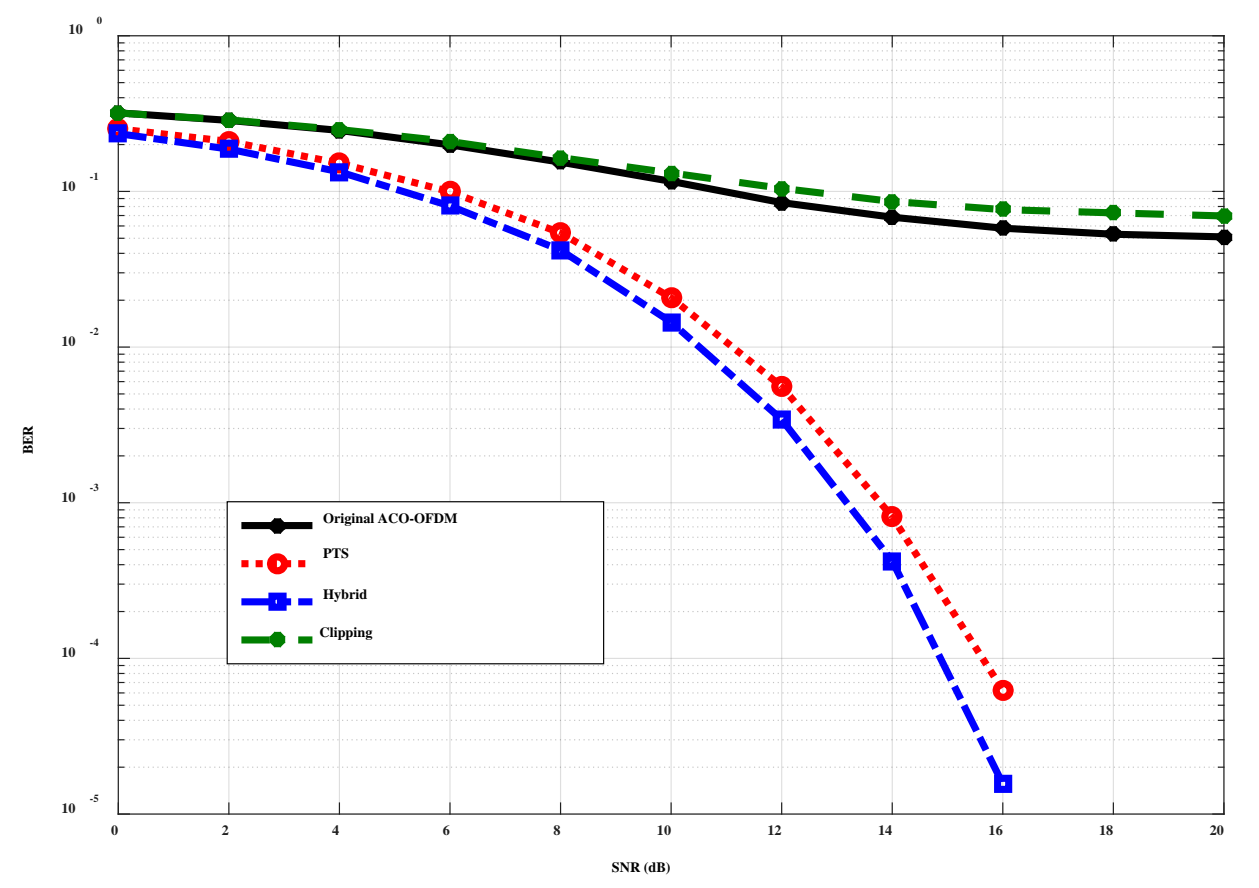

Fig. 6. BER vs. SNR of the ACO-OFDM signal with different methods

The BER performance of the proposed system in OWC channel with AWGN is assessed and compared with other approaches as depicted in Fig. 6. The proposed hybrid scheme provides better BER performance and significantly outperforms all others methods for all SNR values. At SNR of $10 \mathrm{~dB}$, the BER values are around $10^{-1.85}, 10^{-1.65}, 10^{-0.88}$, and $10^{-0.93}$ for the hybrid scheme, modified PTS, clipping method and original ACO-OFDM signals, respectively. The hybrid scheme of ACO-OFDM scheme outperforms the modified PTS method with a $10^{0.2}$ difference in BER which is the closest to the proposed approach. Not surprisingly, the system with signal clipping case shows worse BER performance. From the analysis above, it can be concluded that the hybrid scheme provides high PAPR reduction and also good BER performance in VLC system that based on ACO-OFDM and outperforms the other methods in terms of both the PAPR reduction and the BER performance.

In order to make a fair comparsion between ACO-OFDM and DCO-OFDM, simulations have been performed with the same size of IFFT and same M-QAM i.e. IFFT size is 64 and 16-QAM is used for signal modulation for both optical OFDM formats. Fig. 7 shows the CCDF comparison of PAPR for ACO-OFDM and DCO-OFDM based VLC system with various methods proposed in this work. However, ACO-OFDM has even higher PAPR than DCO-OFDM which is the main shortcoming. The curves show that DCO-OFDM has a lower PAPR as compared to ACO-OFDM system when using the same method. Clearly, the hybrid scheme achieves higher PAPR reduction for both cases as compared to other methods. However, the proposed hybrid scheme for ACO-OFDM has a slightly lower PAPR $(0.1 \mathrm{~dB}$ reduction gain) as compared to DCO-OFDM around CCDF of $10^{-1}$. Also, DCO-OFDM achieves better than ACO-OFDM in terms of PAPR reduction for the corresponding PTS and clipping methods. As shown in Fig. 7, the ACO-OFDM scheme offers approximately 1 and 5 $\mathrm{dB}$ reduction gains in PAPR by using the PTS and clipping methods respectively. 


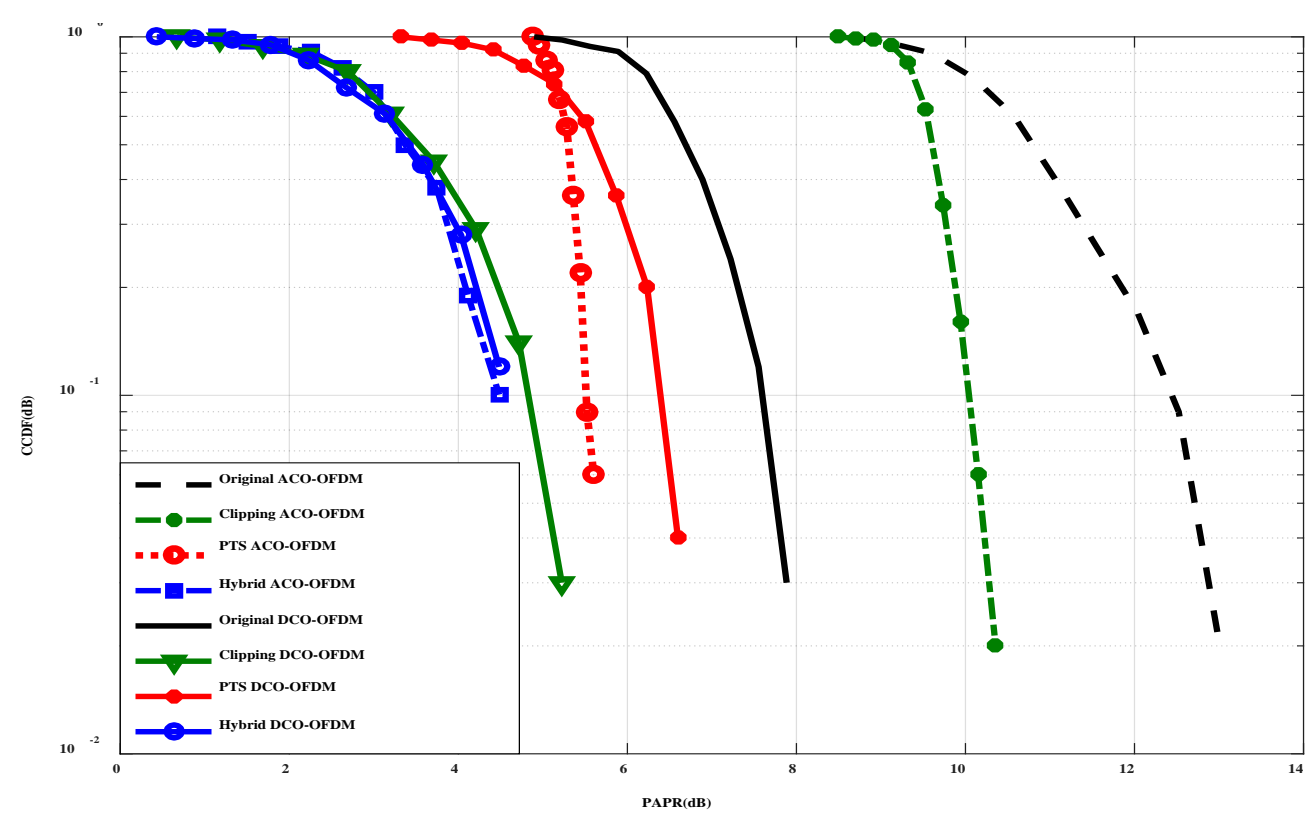

Fig. 7. The CCDF comparison of ACO-OFDM and DCO-OFDM with different approaches

The BER performance of ACO-OFDM and DCO-OFDM versus SNR is shown in Fig. 8. It can be seen that the original ACO-OFDM signal has a better BER performance in compare with the original DCO-OFDM signal at lower SNR values due to its power efficiency. Meanwhile, for higher SNR values (beyond $13 \mathrm{~dB}$ in Figure 8), DCO-OFDM outperforms ACO-OFDM because of spectral efficiency. In other words, ACO-OFDM scheme has a lower SNR requirement as compared to DCO-OFDM at low information rate targets and higher SNR requirement at high information rate targets.

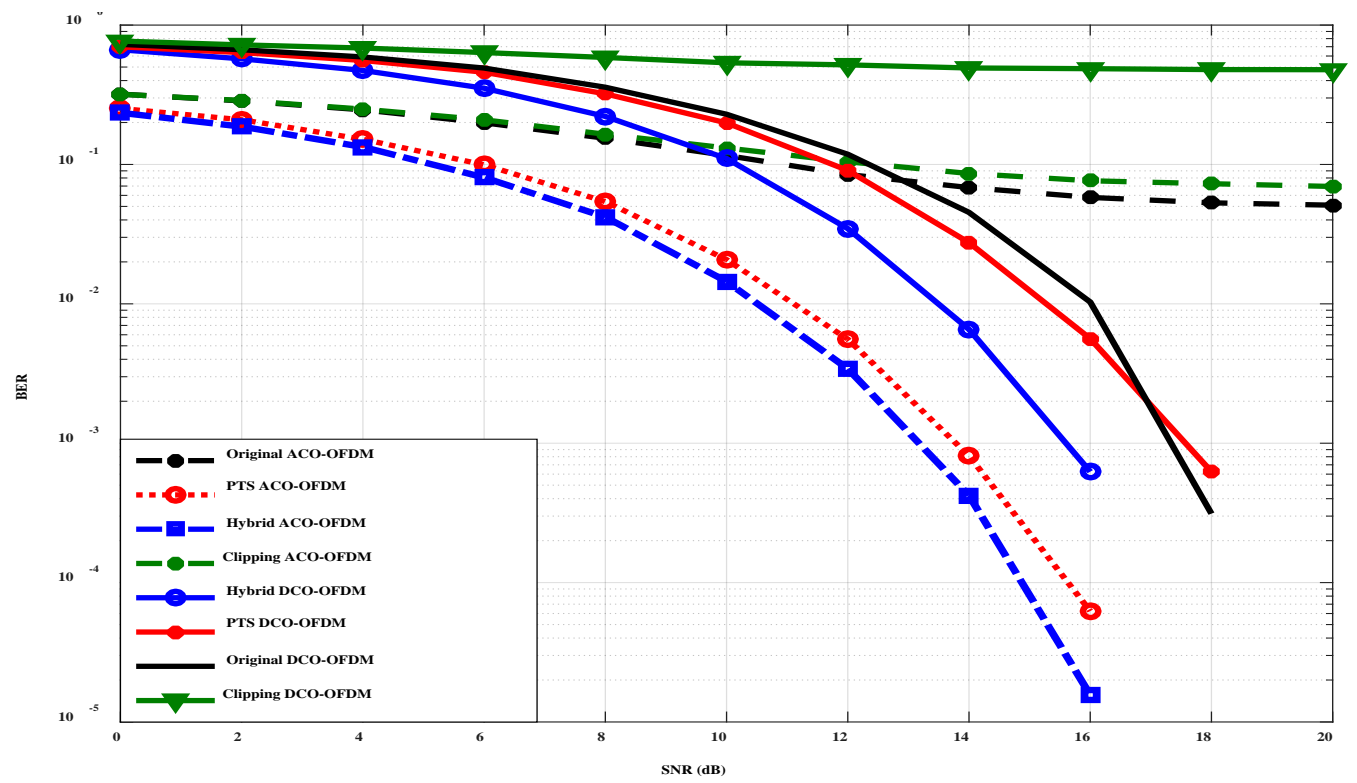

Fig. 8. Comparison of BER performance of ACO-OFDM and DCO-OFDM with different approaches 
Moreover, ACO-OFDM outperforms DCO-OFDM in terms of BER performance for each corresponding method while the latter has a much lower PAPR as compared to ACO-OFDM system when using the same method. For example, ACO-OFDM has approximately $2.5 \mathrm{~dB}$ better performances than DCO-OFDM at BER of $10^{-3}$ for the PTS and hybrid schemes. Furthermore, it can be observed that ACO-OFDM shows a better BER performance than DCO-OFDM for a corresponding clipping scenario over the entire SNR region. However, these improvments in BER performance are achieved at the expense of $50 \%$ reduction in spectral efficiency. The BER of both OFDM formats decreases by increasing the SNR until it reaches an optimum point, and increases afterwards due to the signal clipping imposed distortion.

\section{Conclusion}

In this paper, a new PAPR reduction method for an optical OFDM-based VLC system is developed. ACO-OFDM and DCO-OFDM transmission schemes are compared in terms of PAPR and error rate performance. Due to its power efficiency, the original ACO-OFDM signal is shown to have a better BER performance in compare with the original DCO-OFDM signal at lower SNR values while for higher SNR values, the DCO-OFDM outperforms ACO-OFDM because of its spectral efficiency. Three methods of reducing the PAPR have been considered, namely, PTS, clipping and hybrid schemes and compared with the original OFDM approaches. As it is expected, signal clipping offers significant PAPR reduction but it has a considerable effect on the BER performance. It is shown that, as the clipping ratio increases, the BER performance is improved, but comparably less reduction in PAPR and vice verse. Thus, this trade-off relationship between these two metrics should be considered when choosing the clipping level in order to achieve better performance of the VLC systems. Numerical results show that the proposed hybrid scheme significantly provides effective PAPR suppression and has reduced the BER of VLC system for both OFDM approaches. For ACO- OFDM based VLC system, a PAPR reduction gain of about $8 \mathrm{~dB}$ can be obtained using the proposed hybrid scheme when compared with the basic ACO- OFDM signal at a CCDF of $10^{-1}$. Comparing with other methods, the PAPR reduction gain of the proposed method is about $5.5 \mathrm{~dB}$ and $1 \mathrm{~dB}$ compare to the signal clipping and the modified PTS method, respectively. The corresponding BER values at $10 \mathrm{~dB}$ SNR are approximately $10^{-1.85}, 10^{-1.65}$, $10^{-0.88}$, and $10^{-0.93}$ for the hybrid scheme, modified PTS, clipping method and original ACO-OFDM signals, respectively. Moreover, at the expense of half spectral efficiency, it can be concluded that ACO-OFDM has about $2.5 \mathrm{~dB}$ better performance than DCO-OFDM at BER of $10^{-3}$ when using the PTS and hybrid schemes while it shows a better BER performance than DCO-OFDM for a corresponding clipping scenario over the entire SNR region. Future works will involve the channel estimation methods for further BER improvement along with development of an experimental testbed by mixing real optical front-end and simulated digital modems to support our simulations.

\section{References}

[1] Markets and Markets, "Visible Light Communication (VLC)/Li-Fi Technology \& Free Space Optics (FSO) Market (2017-2022)," tech. rep., 2017.Article (CrossRef Link).

[2] Jean, Armstrong, “OFDM for optical communications,” Journal of Lightwave Technology, Vol. 27, No.3, pp.189-204, 2009. Article (CrossRef Link). 
[3] Dimitrov S., Sinanovic S. and Haas H, "Clipping Noise in OFDM-Based Optical Wireless Communication Systems,” IEEE Trans. on Communications, Vol. 60, No. 4, pp. 1072-1081, 2012. Article (CrossRef Link).

[4] Hussein, Y.S., Alias, M.Y. and Abdulkafi, A.A., "An Implementation of Indoor Visible Light Communication System Using Simulink," in Proc. of IEEE Conf. on Platform Technology and Service (PlatCon), pp. 1-6, February 13-15, 2017. Article (CrossRef Link).

[5] Abdulkafi, A.A., Alias, M.Y. and Hussein, Y.S., "A Novel Approach for PAPR Reduction in OFDM-Based Visible Light Communications," in Proc. of IEEE Int. Conf. on Platform Technology and Service (PlatCon), pp. 1-4, February 13-15, 2017. Article (CrossRef Link).

[6] S. K. Wilson and J. Armstrong, "Transmitter and receiver methods for improving asymmetrically-clipped optical OFDM," IEEE Transactions on Communications, Vol.8, No.9, pp.4561-4567, 2012. Article (CrossRef Link).

[7] W. Xu, M. Wu, H. Zhang, X. You, and C. Zhao, "ACO-OFDM-specified recoverable upper clipping with efficient detection for optical wireless communications," IEEE Photonics Journal, Vol. 6, No. 5, pp.1-17, 2014. Article (CrossRef Link).

[8] J. G. Doblado, A. C. Oria, V. B. Lecuyer, P. Lopez, and D. P. Calderon, "Cubic Metric Reduction for DCO-OFDM Visible Light Communication Systems," Journal of Lightwave Technology, Vol. 33, No. 10, pp.1971-1978, 2015. Article (CrossRef Link).

[9] B. Ranjha, M. Kavehrad, "Precoding techniques for PAPR reduction in asymmetrically clipped OFDM based optical wireless system," SPIE OPTO. International Society for Optics and Photonics, Vol. 8645, No.6, pp. 540-547, 2013. Article (CrossRef Link).

[10] B. W. Kim and S. Y. Jung, "Bandwidth-efficient Precoding Scheme with Flicker Mitigation for OFDM-based Visible Light Communications," ETRI Journal, Vol. 37, No. 2, pp. 1-7, 2015. Article (CrossRef Link)

[11] Kang, Weiwei, and Steve Hranilovic, "Power reduction techniques for multiple-subcarrier modulated diffuse wireless optical channels," IEEE Transactions on Communications, Vol. 56, No. 2, pp.279-288, 2008. Article (CrossRef Link)

[12] M. Z. Farooqui, P. Saengudomlert, and S. Kaiser, "Average transmit power reduction in OFDM-based indoor wireless optical communications using SLM," in Proc. of International Conference on Electrical and Computer Engineering, pp. 602-605, September 8-10 2010. Article (CrossRef Link).

[13] Nadal, L. , Michela S. M., Josep M. F., and Gabriel J., "Low complexity bit rate variable transponders based on optical OFDM with PAPR reduction capabilities," in Proc. of IEEE European Conference on Networks and Optical Communications, pp. 1-6, 2012. Article (CrossRef Link).

[14] Hou, J., Zhao X., and Fei Hui, "Reduction of Peak-to-Average Power Ratio of Multicarrier Modulation Signals with Adaptive Companding Scheme," KSII Transactions on Internet \& Information Systems, Vol. 10, no. 7, pp. 3117-3130, July 2016. Article (CrossRef Link).

[15] N. Bibi, A. Kleerekoper, N. Muhammad, B. Cheetham, "Equation-method for correcting clipping errors in OFDM signals," SpringerPlus, Vol. 5, No. 1, pp.1-11, 2016. Article (CrossRef Link).

[16] Elhelw, A.M. and Badran, E.F., "Semi-Blind Error Resilient SLM for PAPR Reduction in OFDM Using Spread Spectrum Codes," PloS one, Vo. 10, No.5, pp. 1-12, 2015. Article (CrossRef Link).

[17] S. H. Muller and J. B. Huber, "OFDM with reduced peak-to-average power ratio by optimum combination of partial transmit sequences," Electronic Letters, Vol. 33, No. 5, pp. 368-369, 1997. Article (CrossRef Link).

[18] Z. Fulai, L. Liu and J. Yang, "DFT-spread combined with PTS method to reduce the PAPR in VLC-OFDM system," in Proc. of IEEE International Conference on Software Engineering and Service Science, pp.629-632, 2014. Article (CrossRef Link).

[19] E. Z. Ankarali, I. H. Syed, M. Abdallah, K. Qaraqe, H. Arslan and H. Haas, "Clipping noise mitigation using partial transmit sequence for optical OFDM systems," IEEE Internatioal Workshop on Optical Wireless Communications, pp. 80-84, 2014. Article (CrossRef Link). 
[20] A. A. Abdulkafi, M. Y. Alias and Y. S. Hussein, "Performance Analysis of DCO-OFDM in VLC System," in Proc. of IEEE Malaysia International Conferenc on Communications, pp. 1-6, 2015. Article (CrossRef Link).

[21] Armstrong J. and Schmidt, "Comparison of asymmetrically clipped optical OFDM and DC-biased optical OFDM in AWGN," IEEE Communications Letter, Vol.12, No.5, pp.343-345, 2008. Article (CrossRef Link).

[22] Y. Cho, S. Yong, J. Kim, W. Y. Yang, and C.G. Kang, "MIMO-OFDM wireless communications with MATLAB," John Wiley \& Sons, 2010. Article (CrossRef Link).

[23] Jeong, G.M., Yeo, J.Y., Won, D.M., Bae, S.H. and Park, K.J., "Design and Implementation of an LED Mood Lighting System Using Personalized Color Sequence Generation," KSII Transactions on Internet \& Information Systems, Vol. 6, No. 12, pp. 3128-3196, December 2012. Article (CrossRef Link).

[24] H. Elgala, R. Mesleh, and H. Haas, "Non-linearity effects and Predistortion in optical OFDM wireless transmission using LEDs," International Journal Ultra Wideband Communication Systems, Vol. 1, No. 2, pp. 143-150, 2009. Article (CrossRef Link) . 

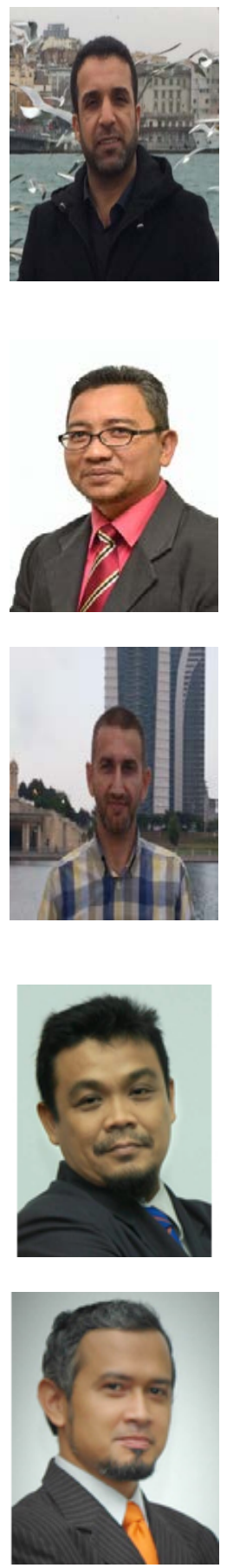

Ayad Atiyah Abdulkafi received the B.Sc. and M.Sc. degrees in Electrical Engineering (major in telecommunications) from Al-Mustansiriya University, Baghdad, Iraq, in 2001 and 2004, respectively. He then received his Ph.D. in Wireless Communication Engineering from University Tenaga Nasional (UNITEN), Malaysia in 2015. He was a postdoctoral research Fellow at faculty of engineering, Multimedia University, Cyberjaya, Malaysia. He is a staff member in College of Engineering, Tikrit University, Iraq. His research interests are in wireless communications, including, LTE, heterogeneous networks, energy-efficient wireless network design, Green Cellular Networks, OFDM, optical wireless communications, Visible Light Communication. Email: al.ayad@yahoo.com.

Mohamad Yusoff Alias obtained the Bachelor of Science in Engineering (Electrical Engineering) degree from the University of Michigan, Ann Arbor, in May 1998. He then received his Ph.D. degree in December 2004 from the School of ECS, University of Southampton in the United Kingdom. He is currently a Professor at the Faculty of Engineering, Multimedia University in Malaysia. His research interests cover the field of wireless communications especially in OFDM, multiple antenna system, multiuser detection, genetic algorithms in communications, multimedia applications and visible light communication.

Yaseein Soubhi Hussein received his BSc degree in Electrical Engineering from University of Baghdad, Iraq, in 1999. His Master degree in Telecommunications Engineering was from University of Malaya, Malaysia, in 2010 and $\mathrm{PhD}$ in Communication and Networks Engineering, Universiti Putra Malaysia in 2014. He is currently a Postdoctoral Research Fellow at Faculty of Engineering, Multimedia University, Cyberjaya, Malaysia. His main research interests are interference coordination, handover, 4G and 5G Mobile Wireless: Long Term Evolution (LTE) and LTE-Advance, Wireless Channel Modeling, Millimeter-Wave (mmWave) and visible light communication (VLC), also known as Li-Fi. E-mail: yaseein@ieee.org, yaseein73@gmail.com, Phone: +60 173204329.

Nazaruddin Omar received his Computer Science degree and Master degree in Computer Science from University Teknologi Malaysia, in 1994 and 2009, respectively. He is currently Principal Researcher at TM Research \& Development, Malaysia. His main research interests are Biometric,Data Security,Sensor Network,Access Control,Visible Light Communication, Software Define Network and Network Functional Visualization.He is currently a technical committee of the ISO/IEC JTC 1/SC 37 Biometrics hosted by SIRIM.He has received two MOSTI Research Grants in 2006.

Kamarulzamin Salleh received his Electrical Engineering degree from University Teknologi Malaysia, Skudai Johor in 2005 and Master of Electrical Engineering from Universiti Teknologi Mara, Shah Alam. Currently he is a Researcher in TM R\&D involving in Visible Light Communication projects. 